\title{
AVALIAÇÃO PRELIMINAR DA TAXA DE SEDIMENTAÇÃO NA REGIÃO DE CULTIVO DE MOLUSCOS (PERNA PERNA) NA ENSEADA DA ARMAÇÃO - SC.
}

\author{
SCHETTINI, C.A.F.; RESGALLA JR., C. \& K.N. KUROSHIMA \\ Faculdade de Ciências do Mar - FACIMAR/UNIVALI
}

\begin{abstract}
RESUMO
A atividade de cultivo do molusco Perna perna (Linnaeus, 1758) vêm crescendo de maneira expressiva ao longo do litoral do Estado de Santa Catarina nos últimos anos. Embora essa atividade apresente aspectos positivos em termos econômicos para as comunidades de pescadores artesanais, ela também apresenta impactos ambientais para as regiões onde se desenvolve. Desses impactos, o aumento da taxa de sedimentação potencial é um dos efeitos comumente observados. A Enseada da Armação de Itapocoroy é um sistema abrigado do ataque de ondas provenientes do quadrante sul, e as correntes devido à maré raramente ultrapassam $0,1 \mathrm{~m} \cdot \mathrm{s}^{-1}$. Durante um período de 51 horas, de 30 de agosto até $1^{\circ}$ de setembro de 1995, foi instalado um sistema de armadilhas de sedimentos abaixo de uma corda de cultivo localizada em uma região com $4 \mathrm{~m}$ de profundidade. $\mathrm{O}$ sistema ficou a $1 \mathrm{~m}$ acima do fundo. Esse sistema de armadilhas consiste de um flutuador com 4 tubos de PVC (armadilhas) com relação comprimento/diâmetro $=5$, sendo colocado e retirado com o auxílio de mergulho autônomo. Em dois dos tubos foram adicionados $100 \mathrm{ml}$ de uma solução de $\mathrm{NaCl} 50$ $\%$, e nos outros dois tubos foram adicionados o mesmo volume de solução com formaldeído $5 \%$. O material retido nas armadilhas foi filtrado em filtro de fibra de vidro (poro $=0,45 \mathrm{gm}$ ), e desalinizado. A partir do material retido nos filtros foram analisados (1) a massa total retida, (2) organismos planctônicos com lupa, (3) carbono orgânico por titulação e (4) matéria orgânica total por combustão em mufla. A taxa de sedimentação potencial foi estimada pela relação massa retida / (tempo de permanência . área da armadilha). Os resultados mostraram uma taxa de sedimentação média de $118 \pm 65 \mathrm{~g} \cdot \mathrm{m}^{-2} \cdot \mathrm{dia}^{-1}$ (peso seco). Da massa retida, $17 \%$ consiste de matéria orgânica total, sendo que dessa matéria orgânica, $24 \%$ é constituído de carbono orgânico, ou $4 \%$ da massa total. Nos filtros foram encontrados zooplâncton apenas nas armadilhas com solução de formaldeído. A taxa de sedimentação potencial encontrada para a Enseada da Armação de Itapocoroy, $118 \pm 65 \mathrm{~g} \cdot \mathrm{m}^{-2}$. $\mathrm{dia}^{-1}$, é superior à taxas encontradas em outras regiões de cultivo ao redor do mundo, no entanto deve ser considerada como uma abordagem inicial.
\end{abstract}

Palavras Chave: Sedimentação, Armadilhas de Sedimento, Aquacultura de Mexilhões.

\section{PRELIMIRARY ASSESMENT ON THE SEDIMENTATION RATE OF THE ARMAÇÃO DE ITAPOCOROY BIGHT MUSSEL CULTURE SITE - SC.}

\begin{abstract}
The mussel culture along the Santa Catarina State has increased during the last few years, and nowadays represents an important activity in complement to fishing. Although this occupation represents a new income source for the fishermen communities, it brings together several environ $\neg$ mental problems. The increase of the potential sedimentation rate is one of these problems. The Armação de Itapocoroy Bight is sheltered from the frontal attack of waves from the south, and tidal currents are usually lower than $0.3 \mathrm{~m} . \mathrm{s}^{-1}$.During a 51 hours period from August 30 to September 1, 1995, a sediment trap system was deployed under a mussel culture long line, in a region $4 \mathrm{~m}$ deep on average. The system was placed $1 \mathrm{~m}$ over the bottom, and consisted of a floatation frame with four PVC tubes (the traps). The traps have the relation length/diameter about 5 , and its deployment and retrieve were made with the help of scuba divers. We added in two traps $100 \mathrm{ml}$ of a $50 \% . \mathrm{NaCl}$ solution, and in the other traps it was added the same solution plus $5 \%$ formaldehyde. The material trapped was filtered throughout fiber glass filters (pore $=0.45 \mathrm{pm}$ ), and had the salt washed out. From
\end{abstract}




\begin{abstract}
the material retained on the filters, we analyzed (1) the total mass, (2) zooplanktonic organisms, (3) organic carbon using standard methods titration, and (4) total organic matter by combustion. The potential sedimentation rate was estimated by the relation retained mass / (deployment time . mouth trap area). The results showed a mean sedinnentation rate of $118 \pm 65 \mathrm{~g} \cdot \mathrm{m}^{-2}$.dia ${ }^{1}$ (dry weight). The trapped material consisted of $17 \%$ of total organic matter, being $24 \%$ of organic carbon, which represented $4 \%$ of the total mass. In the traps with formaldehyde solution it was found a greater abundance of zooplankters. The estimated potential sedimentation rate for the Armação do Itapocoroy Bight, $118 \pm 65 \mathrm{~g} \cdot \mathrm{m}^{-2} \cdot \mathrm{dia}^{-1}$, was greater than the values found for other mussel cultures around the world, but must be considered as an initial approach only.
\end{abstract}

Keywords: Sedimentation, Sediment-traps, Mussel Aquaculture.

\section{INTRODUÇÃO}

A atividade de cultivo de moluscos bivalves marinhos vêm se desenvolvendo de uma maneira bastante intensa ao redor do mundo. Esse tipo de atividade causa inevitáveis impactos ambientais, e dependendo de como esse tipo de cultura é implementada, os danos ao ambiente podem se tornar demasiadamente pesados. Os problemas que surgem com o aumento da população de organismos filtradores são o aumento da taxa de sedimentação potencial, a alteração do regime de correntes, e o incremento do fluxo vertical de carbono.

Em muitos locais já existem estudos sobre o impacto que essa atividade causa nos ambientes costeiros, observando-se em todos os casos o aumento da taxa de sedimentação efetiva e potencial. Entendese que taxa de sedimentação potencial é o fluxo vertical de sedimentos, medido com o uso de armadilhas de sedimento. Este fluxo não representa a sedimentação efetiva que é aquela onde o sedimento é incorporado no registro geológico, avaliada por técnicas isotópicas, e.g., chumbo 210.

Os sedimentos finos gerados pelo intemperisnno continental são transportados pelos rios para a zona costeira onde sofrem inúmeros processos de sedimentação e remobilização até ficarem retidos em definitivo no registro geológico (Wright \& Nittrouer, 1995), fazendo com que a sedimentação efetiva possa ser ordens de grandeza inferior à sedimentação potencial observada (ten Brinke et al., 1995).
As regiões de cultivo de mexilhões atuam diretamente na compactação do material particulado em suspensão, e indiretamente aumentando a fricção do meio acarretando a diminuição da velocidade de escoamento. O hábito alimentar dos moluscos cultivados faz com que eles atuem como empacotadores de material em suspensão. Durante esse processo, o animal ingere indiscriminadamente todas as partículas que estão em suspensão na água, assimilando somente as de natureza orgânica.

Os sedimentos que estão em suspensão permanecem nesse estado por estarem em um equilíbrio entre o seu volume específico e o grau de turbulência do meio (Komar, 1976). Quando esses sedimentos são ingeridos por um organismo filtrador, eles são empacotados e cimentados com muco ao passar pelo sistema digestivo e expelidos novamente para o meio. Dessa forma, ocorre um ganho do volume específico o que impede que esse pacote de sedimentos e matéria orgânica permaneça em suspensão por muito tempo, havendo um considerável incremento da velocidade de sedimentação.

Esses pacotes são denominados de fezes ou pseudo-fezes. A diferença entre eles reside no fato de que quando existe abundância de material em suspensão para ingestão do organismo, após um período onde ocorre a ingestão, assimilação e liberação de parte da suspensão (fezes), o organismo permanece filtrando o meio mesmo sem que haja assimilação, ocorrendo apenas o empacotamento do sedimento em suspensão (pseudo-fezes), e o enriquecimento do conteúdo de matéria 
orgânica pelo muco.

O objetivo do presente estudo foi avaliar a metodologia de armadilhas de sedimento com o propósito de se estimar fluxos verticais em zonas de cultivo de moluscos bivalves. Devido a grande importância do meio ambiente para o desenvolvimento do turismo no Estado de Santa Catarina, o desenvolvimento de tecnologias de baixo custo para avaliação de impactos causados pela atividade de cultivo de moluscos bivalves provê ferramentas para quantificação de tais impactos, possibilitando seu controle.

\section{AREA DE ESTUDO}

A atividade de cultivo de moluscos marinhos vêm se desenvolvendo intensamente ao longo do Estado de Santa Catarina devido as condições fisiográficas favoráveis da sua costa. Apesar de ser uma atividade recente na região, ela já está atingindo valores significativos em termos de produção e representa uma alternativa econômica para as comunidades de pescadores artesanais da região. A produção na Enseada da Armação de Itapocoroy (Fig. 1) aumentou de 190 toneladas em 1990 para 5.000 toneladas em 1995, o que atualmente representa $10 \%$ da produção estadual (Manzoni \& Lavaca, 1996).

A Enseada da Armação é uma ria com elevações de até 250 metros de altitude, porém as profundidades não chegam à 15 metros. Esta enseada é uma região abrigada das ondas e ventos provenientes do quadrante sul, sendo um abrigo natural para embarcações. Devido à essa geografia favorável, está se desenvolvendo nesse local um centro emergente de cultivo de moluscos no estado, e onde se desenvolve as atividades de pesquisa do Laboratório de Maricultura da Faculdade de Ciências do Mar da Universidade do Vale do Itajaí.

Os ventos predominantes na região são de NE durante a maior parte do tempo, com alteração desse quadro durante o inver- no, quando ocorre uma intensificação dos ventos de sul com a passagem de sistemas frontais. A maré astronômica local apresenta uma altura média de 0,86 $\mathrm{m}$, com máximas de 1,10 me mínimas de $0,24 \mathrm{~m}$. O padrão semi-diurno com desigualdade de altura para preamares e baixamares consecutivas, e a razão de forma dada pela soma dos principais constituintes harmônicos diurnos sobre os principais constituintes semi-diurnos $\left(\mathrm{O}_{1}+\mathrm{K}_{1}\right) /\left(\mathrm{M}_{2}+\mathrm{S}_{2}\right)$ (Pond \& Pickard,1989) é 0,4 .

As correntes na região raramente ultrapassam $0,1 \mathrm{~m} \cdot \mathrm{s}^{-1}$, sendo influenciadas grandemente pelo vento local. Observações horárias realizadas durante sete dias com correntógrafos em outubro 1994 indicaram um sentido de corrente residual de ENE na região de cultivo sob condições de ventos de NE. A salinidade na enseada está diretamente relacionada com as condições hidrológicas do estuário do Rio Itajaí-açu e das massas de água predominantes sobre a plataforma continental adjacente. Isso faz com que existam abruptas oscilações da salinidade e temperatura como resposta eventos meteorológicos.

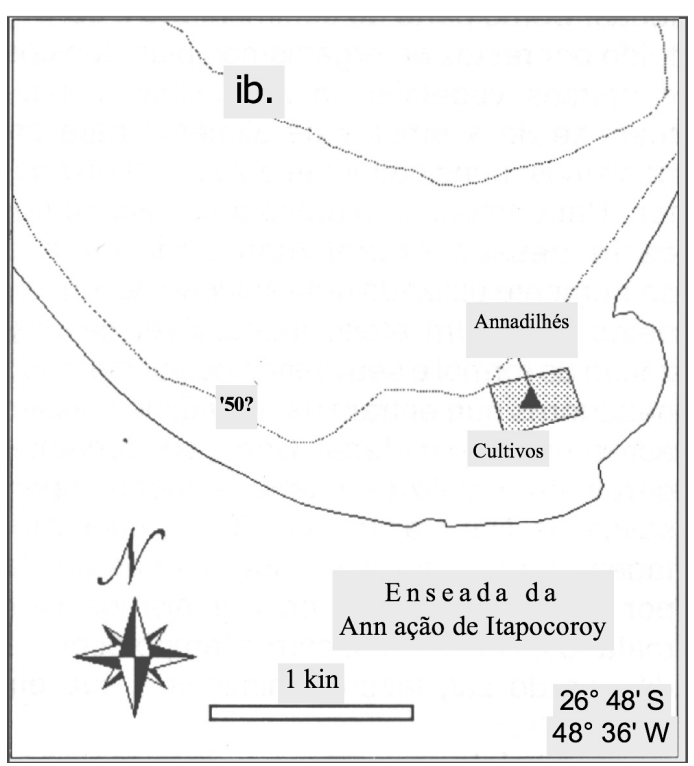

Figura 1 - Área de estudo com a localização das zonas de cultivo. 


\section{MATERIAL E MÉTODOS}

O princípio da metodologia de armadilhas de sedimento no estudo de fluxos verticais reside em criar um micro ambiente onde não exista condições para manutenção de partículas em suspensão, ocasionando então a sedimentação em uma área e um tempo conhecidos. Apesar de existirem muitos formatos diferentes para armadilhas de sedimento, existe um consenso que uma relação entre o comprimento da armadilha e o diâmetro da boca não deve ser inferior â 5 (Gundersen, 1990), embora a eficiência de captura do material em suspensão seja grandemente influenciada pela velocidade horizontal na boca da armadilha e pela velocidade de sedimentação das partículas. Quanto maior for a velocidade horizontal, menor será a eficiência da armadilha (Baker, 1988).

Nos casos de emprego de armadilhas de sedimento por períodos prolongados, ex. mais de alguns dias, existe a necessidade de adicionar conservantes e/ou venenos para evitar a atividade do zooplancton e de bactérias. Como parte do sedimentado é constituído por restos de organismos planctônicos e detritos vegetais, as armadilhas constituem-se de acúmulos de alimento para organismos, principalmente o zoo e ictioplâncton. Para amenizar o efeito do consumo por parte desses organismos nadadores é comumente utilizado uma solução de formaldeído $5 \%$. Um efeito indesejável da utilização do formol é seu efeito nocivo, matando nadadores que entram na armadilha e assim acrescentando material. Como conservante, geralmente utiliza-se uma solução hipersalina de $\mathrm{NaCl}$ acima de $50 \%$, cuja vantagem sobre o formol é que apenas afasta por choque osmótico os organismos sem mata-los. No entanto, com o tempo ocorre a difusão do sal, fazendo diminuir a sua eficiência.

A coleta para esse estudo foi realizada com um sistema flutuador com quatro armadilhas de sedimentos com relação comprimento: diâmetro $=5$, modificado de Larsson

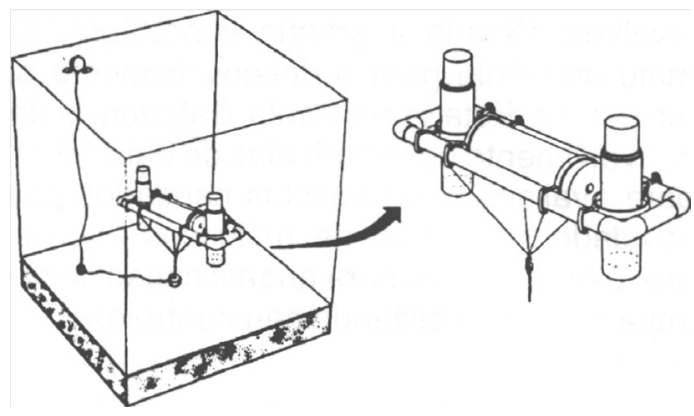

Figura 2 - Sistema flutuante de armadilhas de sedimento (Larsson et al., 1986).

et al. (1986; Fig. 2). O período amostral foi de 30 de agosto até 1 de setembro de 1995, com um total de 51 horas de permanência das armadilhas na água (aproximadamente 4 ciclos de maré). $O$ sistema foi posicionado abaixo de uma corda de moluscos, flutuando â Im acima do fundo em uma região de 3,5 m de profundidade na baixamar.

As armadilhas foram colocadas cuidadosamente com mergulho autônomo. Em duas das armadilhas foram adicionados 100 $\mathrm{ml}$ de solução de $\mathrm{NaCl}$, com concentração de $50 \%$. Nas outras duas foram adicionados o mesmo volume da solução salina com formaldeído de concentração $5 \%$. Essas operações foram realizadas com as armadilhas já posicionadas utilizando-se uma seringa de $300 \mathrm{ml}$ e um duo para que o material fosse despejado diretamente no fundo dos tubos.

As armadilhas foram recuperadas utilizando também de mergulho autônomo, tampando-as ainda no fundo antes de removê-las. Em laboratório o material retido foi acondicionado em recipientes de vidros e mantidos refrigerados até o seu processamento. As amostras foram filtradas em filtros Millipore GFC de poro médio 0,45 um. Devido â grande quantidade de material, foram utilizados diversos filtros para cada amostras, 
permitindo realizar análises destrutivas sem a necessidade de se cortar os filtros.

A taxa de sedimentação Ts foi estimada por

$$
T s=\frac{m}{t \cdot A}
$$

onde $\mathrm{m}$ é a massa de material retida da armadilha, té o tempo de permanência na armadilha na água, e Aé a área da boca da armadilha. As unidades utilizadas foram g para a massa, dia para o tempo e $\mathrm{m}^{2}$ para a área. $\mathrm{O}$ resultados são mostrados na tabela 1 . Aárea da boca das armadilhas é $A=1,52 \cdot 10^{-3} \mathrm{~m}^{2}$, e o tempo $t=2,12$ dias.

Os teores de carbono orgânico par-ticulado (COP) foram medidos segundo os métodos padrões de oxidação (Strickland \& Parsons, 1972). A matéria orgânica total foi obtida pela volatilização em mufla a $700{ }^{\circ} \mathrm{C}$ durante 24 horas. A análise de organismos zooplanctônicos foi feita sobre o material já filtrado.

\section{RESULTADOS E DISCUSSÃO}

Os resultados da taxa de sedimentação potencial estão sumariados na Tabela 1. Esses resultados demonstram as armadilhas apresentam uma boa replicabilidade, principalmente para as armadilhas que tiveram o tratamento apenas com solução salina. É provável que o desvio padrão encontrado para as armadilhas com tratamento de sal mais formol esteja associado mais com problemas de manipulação durante a retirada dos tubos do quecom erros causados durante as demais fases da manipulação das amostras.

Um problema encontrado durante a filtração foi que devido a grande quantidade de material, o filtro colmatava rapidamente, fazendo que o processo levasse várias horas para se completar. $\mathrm{O}$ desenvolvimento de uma metodologia de quarteamento das amostras deve sanar esse problema, possibilitando ainda obter sub-amostras significativas para a realização de outras análise sobre o material.

Os resultados da análise de microscopia mostraram que as armadilhas com formol retiveram um número maior de organismos planctônicos (Tabela 2). Isto confirma que as armadilhas estão sujeitas a visitas constantes. Os organismos bentônicos encontrados provavelmente estão associados com queda das linhas de cultivo ou ainda com processos de ressuspensão do sedimento de fundo. Entretanto, devido a grande massa de material retido nas armadilhas, e em função do resultado similar de ambos os tratamentos, acredita-se que o consumo ou retenção de organismos vivos dentro das armadilhas não interfiram de maneira significativa no resultado final. É provável que as interferências causadas por atividade biológicas só sejam verificadas em períodos prolongados de permanência das armadilhas no ambiente.

A taxa de sedimentação potencial encontrada foi relativamente grande quando comparada com estudos em outros locais que utilizaram metodologias similares (tabela 3). A dinâmica sedimentar costeira influencia grandemente os resultados, sendo que em locais onde não existe um aporte fluvial con-

Tabela 1 - Massa média de sedimento retida nas armadilhas (M), materia orgânica (MO), carbono orgânico particulado (COP), e taxa de sedimentação potencial (Ts) para os diferentes tratamentos de conservação das amostras.

\begin{tabular}{lcccc}
\hline \hline Tratamento & $\mathrm{M}(\mathrm{g})$ & $\mathrm{MO}(\%)$ & $\mathrm{COP}(\%)$ & $\mathrm{T}_{\mathrm{s}}\left(\mathrm{g} \cdot \mathrm{m}^{-2} \cdot \mathrm{dia}^{-1}\right)$ \\
\hline $\mathrm{NaCl} 50 \%$. & $0,39 \pm 0,01$ & 16,7 & $4,1 \pm 0,8$ & 121 \\
$\mathrm{NaCl} 50 \%$. Formol & $0,38 \pm 0,30$ & 17,5 & $3,1 \pm 0,6$ & 118 \\
\hline
\end{tabular}


siderável, como em na enseada de Kiel, a taxa de sedimentação potencial foi bastante inferior (Peinert et al., 1982). No entanto, quando são comparados os valores encontrados nesses ambientes de menor dinâmica sedimentar com taxas obtidas para o oceano profundo (Iseki, 1981), fica evidenciado que o ciclo de deposição e remobilização de material sedimentar é mais intenso conforme nos aproximamos da costa.

Antes que o material sedimentar fique retido definitivamente no registro geológico, ele pode participar inúmeras vezes do ciclo de erosão e deposição. Isto é facilmente visualizado com os resultados obtidos por Lund-Hansen et al. (1993), na avaliação comparativa entre o método de armadilhas de sedimento e o método de datação isotópica de chumbo 210. Com o emprego de armadiIhas é possível verificar o fluxo vertical com sentido para baixo que ocorre no ambiente durante o período amostral,mas isso não exclui a possibilidade de que exista um fluxo vertical com sentido para cima. Para o sistema estudado por LundHansen et al. (1983), em uma baia no Mar Báltico, a taxa de sedimentação efetiva estimada por um método mais caro e de difícil acesso (chumbo-210) foi duas ordens de grandeza inferior que a taxa de sedimen-tação potencial estimada por armadilhas de sedimento. Entretanto, isto não inviabiliza a utilização de armadilhas para a determinação da dinâmica sedimentar local.

Como o interesse é investigar a influência de sítios de cultivo de moluscos na dinâmica sedimentar, é possível avaliar o efeito do cultivo em comparação com uma área de referência fora da zona de sua influência. Grant et aL (1995) através do monitoramento semanal com armadilhas de sedimento obteve uma diferença significativa com valores de sedimentação potencial sob os cultivos sendo $60 \%$ superiores que os valores para uma estação de referência.

Tabela 2 - Número de organismos planctônicos e bentônicos observados nas armadilhas com tratamento com formol + sal $(F)$ e somente sal (S).

\begin{tabular}{llrrrr}
\hline Grupos & & F1 & F2 & Si & S2 \\
\hline Planctônico & Copepoda Calanoida & 6 & 12 & 1 & \\
& Copepoda Cyclopoida & & & & \\
& Total & 6 & 12 & 1 & \\
Bentônico & Amphipoda Gammaridea & & 2 & & 1 \\
& Amphipoda Caprellidea & & & & 2 \\
& Ostracoda & & 2 & 0 & 1 \\
& Total & 0 & 2 &
\end{tabular}

Tabela 3 - Comparação da taxa de sediementação Ts em ambientes diferentes.

\begin{tabular}{lcccc}
\hline Local & Ts (g.m-2.dia-1) & Ambiente & Técnica & Fonte \\
\hline Oceano Pacífico Norte & 0,043 & oceânico & armadilhas & Iseki, 1981 \\
Kiel Bight (Alemanha) & $<10$ & costeiro & armadilhas & Peinert et al., 1982 \\
Aarthus Bay (Mar Báltico) & 114,8 & costeiro & armadilhas & Lund-Hansen et aL, 1993 \\
Aarthus Bay (Mar Báltico) & 2,5 & costeiro & chumbo 210 & Lund- Hansen et al., 1993 \\
Upper South Cove (Canadá) & $88,7 \pm 55,6$ & sob cultivo & armadilhas & Grant et al., 1995 \\
Upper South Cove (Canadá) & $36,4 \pm 25,5$ & referência & armadilhas & Grant et at, 1995 \\
Armação de Itapocoroy (SC) & $118 \pm 65$ & sob cultivo & armadilhas & Presente estudo \\
\hline
\end{tabular}


Considerando que em ambas as áreas os processos de ressuspensão foram semelhantes, a proporção obtida evidencia o impacto causado por essa atividade em ecossistemas costeiros. No entanto, ressalta-se que os sedimentos sob os cultivos apresentam uma tendência a diminuir a sua granulometria com o tempo como resultado da sedimentação das fezes e pseudo-fezes, e dessa maneira esses sedimentos estariam mais suscetíveis à remobilização, mas ainda assim como reflexo da atividade de cultivo.

O presente estudo consistiu de apenas uma campanha amostral, e não houve um acompanhamento com uma estação de referência. Mesmo assim, os resultados foram considerados positivos em termos da avaliação metodológica, e darão embasamento para futuros estudos sobre o mesmo tema na Enseada da Armação de Itapocoroy, bem como em outras regiões do Estado de Santa Catarina onde a atividade de cultivo de moluscos vêm crescendo. $O$ valor de sedimentação potencial encontrado de 118 + $65 \mathrm{~g} \cdot \mathrm{m}^{-2}$. dia ${ }^{-1}$ foi relativamente grande, sendo que a obtenção de um valor médio significativo deverá considerar um acompanhamento periódico.

\section{AGRADECIMENTOS}

Os aufores agradecem à coloboração prestada pelos professores Adriano Marenzi e Gilberto Manzoni e demais membros da equipe do Laboratório de Aquicultura da UNIVALI. O presente trabalho foi apresentado como painel na $3^{\circ}$ Reunião Especial da SBPC (Schettini, etal., 1996).

\section{REFERÊNCIAS BIBLIOGRÁFICAS}

Baker, E.T.; Milburn, H.B. \& D.A.Tennant. 1988. Field assessment of sediment trap efficiency under varying flow conditions. J. Mar. Res. 46:573-592. ten Brinke, W.B.M.; Augustinus, P.G.E.F. \& G.W. Berger. 1995. Fine-grained sediment deposition on mussel beds in the Oosterschelde (The Netherlands), determined from echosoundings, radioisotopes and biodeposition field experiments. Estuar. Coast. Shelf Sci. 40:195-217.

Grant, J.; Hatcher, A.; Scott, D.B.; Pocklington, P.; Schafer, C.T. \& G.V. Winters. 1995. A multidisciplinary approach to evaluating impacts of shellfish aquaculture on benthic communities. Estuaries. 18(1A):124-144.

Gundersen, K. 1990. Sampling precision and preservation of sediment trap material. p.6-35. In: D. Wassmann, A.S. Heiskznen \& O. Lindahl [eds.]. Proceeding of the Symposium Sediment trap studies in the nordic countries, 2, Kristineberg Marine Biological Station

Iseki, K. 1981. Particulate organic matter transport to the deep sea by salp fecal pellets. Mar. Ecol. 5:55-60.

Komar, P.D. 1976. Bondary layer flow under steady unidirecional currents.p91-106. In: D.J. Stanley \& D.J.P. Swift [Eds.], Marine sediment transport and environmental management. New York, John Wiley \& Sons.

Larsson, U.; Blomqvist, S. \& B. Abrahamsson. 1986. A new sediment trap system. Mar. Ecol. Frog. Ser.31:205-207.

Lund-Hansen, L.C.; Pejrup, M.; Valeur, J. \& A. Jensen. 1993. Gross sedimentation rates in the North Sea-Baltic Sea transition: effects of stratification, wind energy transfer, and resuspension. Oceanologica Acta. 16(3):205-212.

Manzoni, G.C. \& L. Lacava. 1996 Sobrevivência dos gastrópodes predadores Thais haemastoma (Linnaeus, 1767) e Cymatium p. parthenopeum (von Salis, 1793) em diferentes tratamentos de cas $\neg$ tigo. p.555. In: Reunião especial da SBPC, 3, Florianópolis, Resumos. 
Peinert, R.; Saure, A.; Stegmann, P.; Stoe $\neg$ mem, C. \& H. Haardt. 1982. Dynamics of primary production and sedimentation in a coastal ecosystem. Netherlands J. Sea Res., 16:276-289.

Pond, S. \& G.L. Pickard. 1989. Introdutory dynamical oceanography. 2nd Ed., New York, Pergamon Press.

Schettini, C.A.F.; Kuroshima, K. \& C. Resgalla Jr. 1996. Avaliação da taxa de sedimentação na região de cultivo de moluscos na Enseada Armação de Itapocoroy. p.407. In: Reunião Especial da SBPC, 3, Florianópolis, Resumos SBPC/ UFSC.

Strickland, J.D.H. \& T.R. Parsons. 1972. A pratical handbook of seawater analysis. Fish. Res. Board Can. 310 pp.

Wright, L.D. \& C.A. Nittrouer. 1995. Dispersal of river sediments in coastal seas: six contrasting cases. Estuaries.

18(3):494-508. 